\title{
Thai Adolescent Depression: Recurrence Prevention in Practice
}

\author{
Chonnakarn Jatchavala, M.D. ${ }^{1}$, Stella Chan, DPhil, DClinPsy, CPsychol, FBPsS ${ }^{2}$ \\ 'Department of Psychiatry, Faculty of Medicine, Prince of Songkla University, Hat Yai, Songkhla 90110, Thailand. \\ ${ }^{2}$ School of Health in Social Science, University of Edinburgh, Doorway 6, Medical Quad, Teviot Place, Edinburgh EH8 9AG, \\ Scotland.
}

Received 10 October 2017 • Accepted 7 December 2017 • Published online 24 May 2018

\section{Abstract:}

This article aims to review the current practice of recurrence prevention and intervention of adolescent depressive disorder in Thailand. In particular, we assess the Clinical Practice Guideline of Major Depressive Disorder for General Practitioners (CPG-MDD-GP) for Thailand, which is now the official guideline for all depressive patients who are children, adolescents, and adults in Thailand. Although this current Thai clinical guideline was developed and derived from the National Institute for Health and Care Excellence (NICE) of the United Kingdom and the recommendations and the practice parameters of the American Academy of Child and Adolescent Psychiatry (AACAP), it differs from these guidelines in a number of ways. Specifically, the main tool for the primary assessment of the Thai CPG-MDD-GP is called 9Q which categorizes the severity and follow-up of depressive symptoms by health care providers in a hospital setting, whereas the NICE guideline for depression in children and adolescents is based mainly on the community setting, and the AACAP parameter assesses patients by a direct interview method. Additionally, the Thai CPG-MDD-GP has no premise intervention for recurrence prevention of depression and lacks details regarding the importance of treatment engagement by family and community.

Keywords: adolescent, depression, recurrence prevention, Thailand 


\section{Introduction}

Depression in young people has been a global health concern in the last decade. According to the National Institute of Mental Health, ${ }^{1}$ around 2.2 million adolescents between 12 and 17 years of age in the United State of America (USA) had at least one major depressive episode in the previous year. A study in Thailand reported that the prevalence of depression in adolescents was $14.9 \%{ }^{2}$

According to the kindling hypothesis, longer episodes of depression can increase the risk for future subsequent episodes. ${ }^{3}$ Recurrent depression does not involve only neurobiological susceptibilities but also life stressors. In particular, the kindling hypothesis described the first episode of depression as associated more with psychosocial stressors than the following episodes which were influenced more by impaired neurobiological factors. ${ }^{4}$

Recurrent depression during adolescence may represent life-long persistent depression that is associated with early manifestations of functional and interpersonal maladjustment. ${ }^{5}$ Hence, clinicians should carefully adjust their practice due to recurrent depression. ${ }^{6}$ However, no study on the prevention of recurrence and intervention of adolescent depression in Thailand has focused on psychological intervention in preventing the recurrence of depressive episodes in adolescents.

Thus, this article review aims to appraise the studies and clinical practice of recurrence prevention and intervention of Thai adolescent depressive disorder. However, this article has the limitation that only articles published in the English and Thai languages were included. The database of the Department of Mental Health of Thailand, Ministry of Public Health ${ }^{7}$ was searched in the Thai language for articles published between 1994 and 2014. The search terms were (1) depression, (2) psychosocial intervention, and (3) adolescent. The electronic search identified 18 potentially relevant titles, but none of them were related to the prevention of recurrent depression in adolescents.
At the present time, only the Thai Clinical Practice Guideline of Major Depressive Disorder for General Practitioner (Thai CPG-MDD-GP) has been officially and widely used as the standard guideline for depression in Thailand. ${ }^{8}$ To be relevant for the socio-economic context of Thailand, the guideline was modified from other standard guidelines including the National Institute for Clinical Excellence (NICE) of the United Kingdom (UK), the American Psychiatric Association: Practice Guideline for the Treatment of Patients with Major Depressive Disorder, and the World Federation of Societies of Biological Psychiatry (WFSBP) Guideline for Biological Treatment of Unipolar Depressive Disorders.

This review article compares and appraises the prevention strategies of recurrence in adolescent depression using the Thai CPG-MDD-GP, National Institute for Health and Care Excellence, ${ }^{9}$ and the American Academy of Child and Adolescent Psychiatry (AACAP): Practice Parameter for the Assessment and Treatment of Children and Adolescents with Depressive Disorders. ${ }^{10}$ Since NICE $^{9}$ and AACAP ${ }^{10}$ were prototypes for the overall service protocol in the Thai CPG-MDD-GP, they influence the clinical practice for the prevention of recurrent depression in children and young people not only in the UK or the USA, but also in Thailand and worldwide.

\section{Thail CPG-MDD-GP}

The Thai CPG-MDD-GP is the current guideline for Thai general practitioners in primary and secondary care units where the capacities are 10 to 500 inpatients covering populations up to more than 10,000 people. ${ }^{8}$ This wide gap of service capacity caused the protocol of management to be very brief and targets principally the pharmacological considerations in depression. However, some Thai university hospitals have psychological interventions for depressive children and adolescents, such as the child and adolescent psychiatric rehabilitation program at Siriraj Hospital (e.g., emotional regulation 
training, social skills and communication training). ${ }^{11}$ These interventions are routinely practiced even though there are financial constraints and a lack of mental health personnel.

The Thai CPG-MDD-GP ${ }^{8}$ characterizes depression in children and adolescents as a special group for treatment using the same management protocol as adulthood depression (Figure 1). However, cognitive behavioral-therapy (CBT) and interpersonal therapy for depressed adolescents
(IPT-A) were recommended as stand-alone interventions for mildly to moderately depressive children and adolescents, while no psychotherapy was specifically mentioned for adulthood depression. Dissimilar to the AACAP, ${ }^{10}$ the Thai CPG-MDD-GP does not give any suggestions regarding the duration, frequency or protocol of psychological interventions.

Diagnosis of major depressive disorder

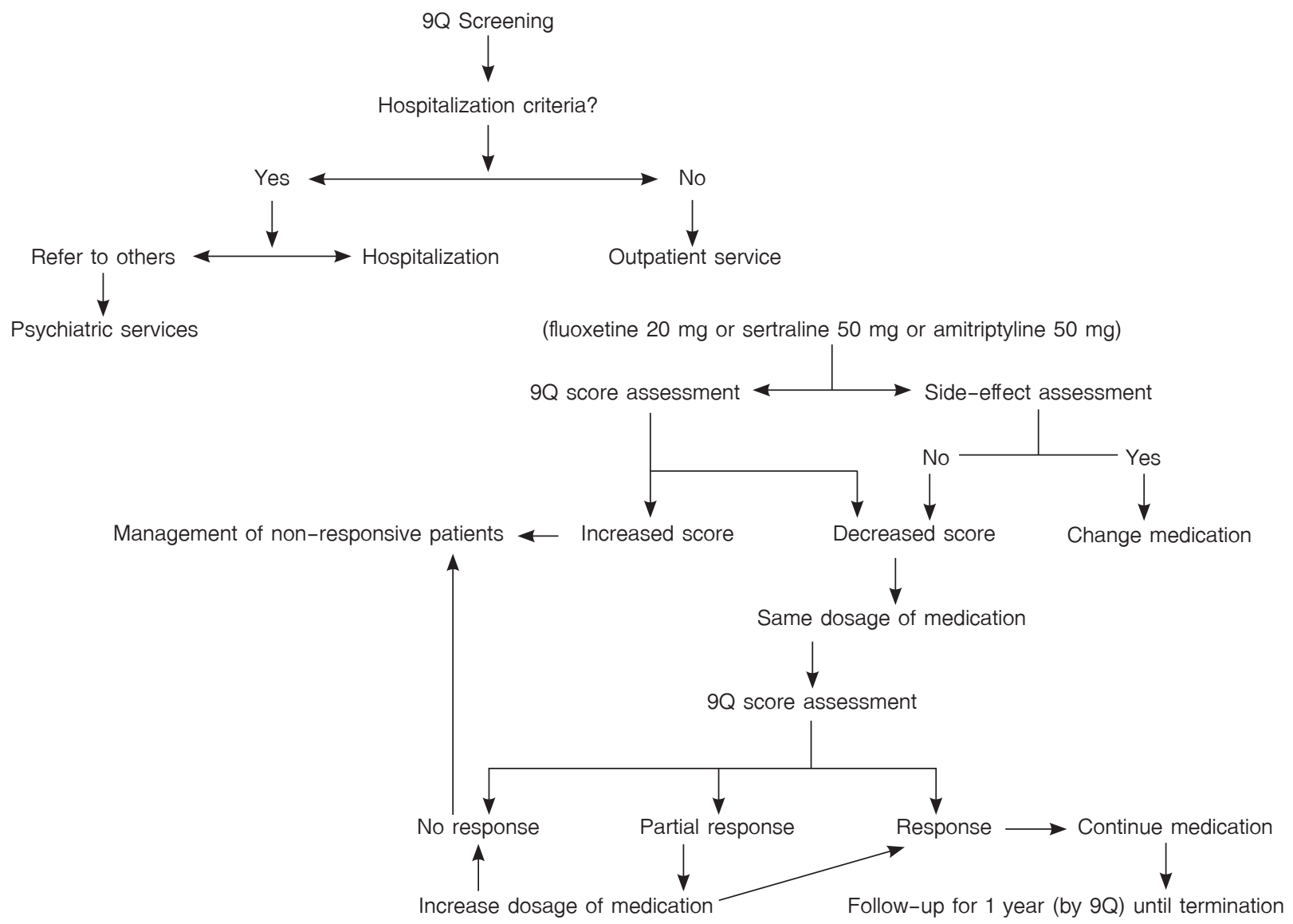

Figure 1 Brief protocol of major depressive disorder in Thailand (Thai CPG-MDD-GP) ${ }^{8}$ 
Even though the guideline is very up-to-date and covers all mainstream pharmacological agents for depression, no psychosocial dimension of management is mentioned in the guideline. The Thai CPG-MDD-GP has no section on the importance of family, school or community engagement in the treatment of depression. No family assessment or adjunctive psychoeducation ${ }^{12}$ is mentioned in this Thai clinical guideline of depression. All responsibilities of depressive disorder care lie with the health care providers.

The primary assessment tool is called the $9 \mathrm{Q}$ or the Thai version of the modified Patient Health Questionnaire. It categorizes the severity of depression using the same categories as in NICE 8 and AACAP 9 (i.e. mild, moderate and severe without psychotic symptoms). ${ }^{13}$ All depressive cases are assessed at the outpatient clinic by health care providers where antidepressants and other medications are prescribed. This is unlike Step 1 of the assessment which $\mathrm{NICE}^{9}$ points out is the responsibility of the personnel in a community context (Table 1). However, in cases of severe depression or psychotic depression, the Thai CPGMDD-GP states that patients should be assessed and intervened at the inpatient unit.

The Thai CPG-MDD-GP indicates that the $9 Q$ is the only tool to assess treatment responses biweekly in the first month and every 4 weeks thereafter until the treatment is terminated. This process is different from the NICE Guideline 8 which points out that self-report questionnaires for depression in young people are to be used for routine screening of children older than 11 years which relates to Child and Adolescent Mental Health Services (CAMHS) at Step 2 (Recognition).

Table 1 Step-care model of depression in children and young people by the National Institute for Health and Care Excellence

\begin{tabular}{|c|c|c|}
\hline Focus & Action & Responsibility \\
\hline Detection & Risk profiling & Tier $1^{*}$ \\
\hline Recognition & Identification in presenting children or young people & Tiers $2^{\star *}-4^{\star * * *}$ \\
\hline \multirow[t]{3}{*}{ Mild depression (including dysthymia) } & Watchful waiting & \\
\hline & Non directive supportive therapy/group cognitive & Tier $1^{*}$ \\
\hline & behavioral therapy/guided self help & Tier $1^{*}$ or $2^{* *}$ \\
\hline \multirow[t]{2}{*}{ Moderate to severe depression } & Brief psychological therapy & Tier $2^{* *}$ or $3^{\star * *}$ \\
\hline & $+/-$ fluoxetine & \\
\hline Depression unresponsive to treatment/recurrent & Intensive psychological therapy & \\
\hline depression/psychotic depression & $\begin{array}{l}+/- \text { fluoxetine, sertraline, citalopram, augmentation with } \\
\quad \text { an antipsychotic }\end{array}$ & Tier $3^{\star * \star}$ or $4^{\star * \star *}$ \\
\hline
\end{tabular}

Tier $1^{*}$ practitioners working in universal services who are not mental health specialists

Tier $2^{* *}$ health-care provider teams in community and primary care settings

Tier $3^{* * *}$ multidisciplinary teams in a community mental health setting or a child and adolescent psychiatry outpatient service

Tier $4^{\star \star * *}$ intensive community treatment services, day units and inpatient units ${ }^{16}$ 
A 9Q score of $<7$ or a decrease of more than $50.0 \%$ would be classified as response-to-treatment cases in the Thai guideline. The clinical conditions of these patients are then followed up for 6 months before treatment termination. Even though the Thai CPG-MDD-GP notifies the healthcare provider for surveillance of a recurrent episode for 12 months following treatment termination, the Thai CPG-MDD-GP does not specify any practice for the prevention of recurrence in either adulthood or adolescent depression.

\section{NICE: depression in children and young people}

Above all, the stepped-care model by NICE states that treatment of depression in children and adolescents should be based in outpatient clinics or the community setting (Table 1). In order to improve long-standing remission and recovery outcomes, mental health care professionals should advise patients and their families of these four recommendations for long-term lifestyle modifications: (1) regular exercise program; (2) sleep hygiene; (3) anxiety management; and (4) a balanced diet. But in practice, NICE does not indicate specific suggestions, such as what lifestyle they should modify, when to begin and when to terminate the modification, where individuals can ask for detailed advice, and how they can modify their lifestyle in a practical way.

Step 1 (detection) states that the responsibilities of pre-recognition belong to the primary health care services, school, and other relevant community settings. These professionals are required to provide active listening and screening skills to provide support and prevent the persistence of symptoms. NICE (2013) recommends that they should record early signs of depression and major life stress for consideration of referral to CAMHS. If young people show any sign of progressive depression, they should be referred for rapid assessment through Step 2 or for recognition by CAMHS as soon as possible. In any case, surveillance for recurrence in the recovery period is not mentioned throughout these steps although the primary services and schools are the main resources of support during the recovery period. For these reasons, primary health care providers and school personnel should play larger roles in both the detection and prevention of occurrence and recurrence of depression. Even though Step 3 acknowledges that it is possible to recover from mild depression without any intervention, the guideline recommends 2 weeks of watchful waiting and that the choices of recommended psychosocial treatments (i.e. group cognitive behavioral-therapy (GCBT) or guided selfhelp) should be discussed between the young people, their clinician, and their families at the first visit (NICE, 2013). Even if the primary focus is to relieve acute depressive symptoms, psychosocial intervention should be considered as part of the ongoing support to maintain symptom reduction and prevent recurrence. Therefore, psychosocial treatment considerations should not only be considered at Step 3 (mild depression) or at Step 4-5 (moderate to severe depression), but emphasis should also be placed on the long-term effectiveness of recurrence prevention strategies.

In the case of moderate to severe depression in adolescents, combined pharmacotherapy with psychosocial treatment is recommended. NICE (2013) specifies that individual CBT, IPT-A, family therapy, and psychodynamic psychotherapy should be offered and discussed in Steps 4 and 5. While the guideline emphasizes the importance of evidence that supports specific pharmacotherapy in youths aged 12-17 years old, NICE (2013) does not mention any recommended particular psychosocial treatment in any age group.

Following remission in Steps 4 and 5, the outcome can be either recovery or recurrent depression. NICE recommends that CAMHS follow cases for 12 months after 
remission. But the 12-month period may not be proper for follow-up in the recovery of depression in every adolescent. ${ }^{14}$ In terms of the recurrence rate, Birmaher et al. ${ }^{15}$ reported $30.0 \%$ of participants reported at least one recurrent depressive episode in up to 24 months following the completed treatment. This finding suggests that it may be more appropriate to follow the cases for a period of 24 months in primary health care as indicated at Step 1 if there is a high risk of recurrence (i.e. early onset of depression, suicide or ADHD).

With respect to the core concept of the NICE guideline for community-based treatment of depression, prevention of recurrence should also be established in the immediate environment of the young people, such as at the school or primary care unit. Psychological intervention should also be based on a time table and life-style to enhance compliance of treatment. According to most previous studies, GCBT as a prevention intervention conducted at school after class may be effective because the service assessment would be convenient. Hence, family members, peers, teachers, and those who live in the relevant community are the most important support system for mutual care. They should be engaged in the treatment not only at Step 1, but also in every step of care including recurrence prevention.

\section{AACAP: Practice Parameter for the Assessment and Treatment of Children and Adolescents with Depressive Disorders ${ }^{9}$}

Compared with the NICE guideline, ${ }^{9}$ the AACAP $^{10}$ allocates more responsibilities for both assessment and treatment of depression to the healthcare providers. The AACAP $^{10}$ states that the routine duty of all involved clinical practitioners (e.g., school nurse, pediatrician, and general practitioner) is to screen depression in every child and adolescent whether they have had a prior depression episode or not. According to cognitive develop- ment in this age group, clinicians should evaluate young people by using the direct interviewing method (face-toface) with the youths and their families.

In the same way as the NICE guideline, ${ }^{9}$ the AACAP differentiates managements according to the severities of depression (i.e. mild, moderate, and severe depressive disorder). In preference to the stepped-care model, the AACAP divides the course of treatment into acute, continuous, and maintenance phases. Regarding AACAP's primary goal, treatment response is defined as the absence of depressive disorder criteria and restoration of normal functioning (Figure 2).

The AACAP emphasizes more importance on psychosocial treatment with 4-6 weeks of supportive therapy (i.e. psychoeducation and case management) being described as the treatment of choice for mild depressive disorder. In the cases of moderate to severe or recurrent depression, the AACAP recommends 6-8 weeks of CBT and interpersonal therapy. Severe depression in children and adolescents requires antidepressant alone or combined with psychotherapy. For example, individual CBT plus antidepressant use in adolescents with mild to moderate depression has been found to lead to a higher rate of remission. In cases of non-response to CBT or in combination with pharmacotherapy, the AACAP suggests that clinicians provide interpersonal and psychodynamic psychotherapy individually.

The AACAP guideline gives more details than the NICE regarding follow-up. The standard goal to achieve remission of depression symptoms is every young person with depressive disorder should be seen by a clinician every week for the first 4 weeks and then biweekly for a total of 12 weeks of treatment. However, the average duration to clinical recovery from the previous studies was 22.8-27.0 weeks. $^{14,15}$ Therefore, a 12-week follow-up duration may not be appropriate to assure remission and recovery of depression. Similar to the NICE guideline, ${ }^{9}$ a 


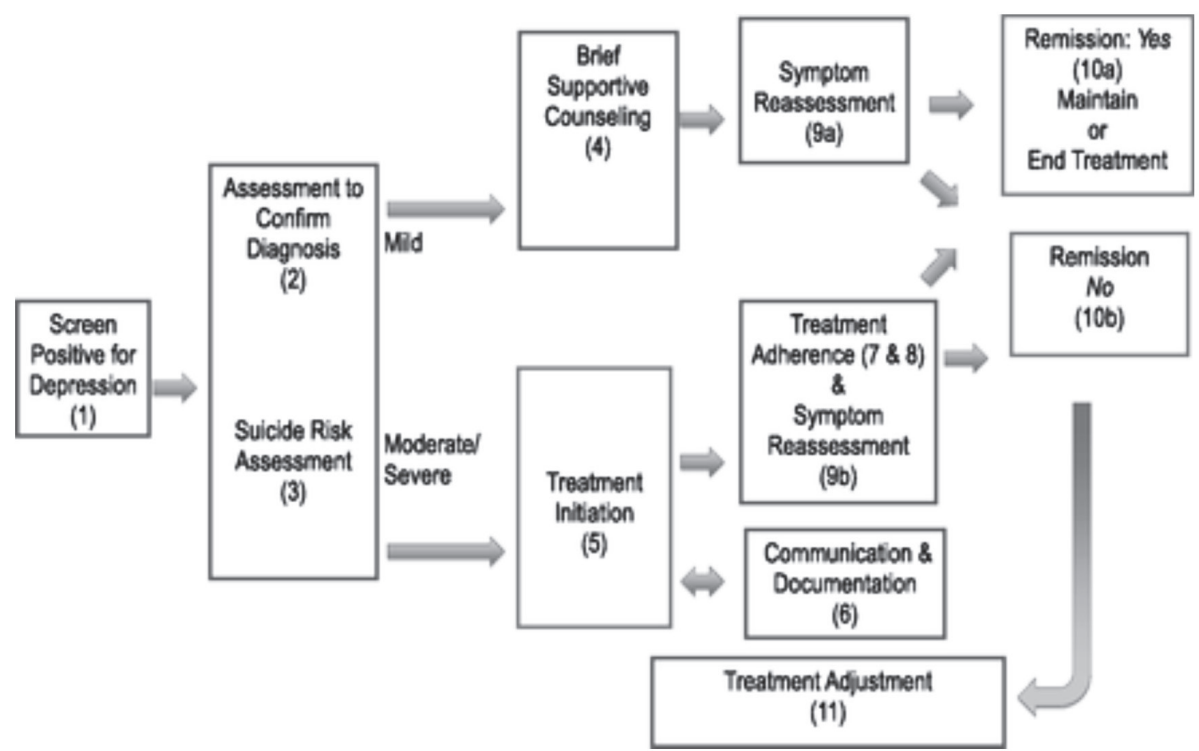

Figure 2 Depression management care pathway based on American Academy of Child and Adolescent Psychiatry ${ }^{17}$

12-month maintenance period may not be sufficient since the average time from remission to recurrent onset of depression was between 16.4 and 26.3 months. ${ }^{6}$

Even though the $\mathrm{AACAP}^{10}$ emphasizes the prevention of recurrent depression in the maintenance phase, no specific psychological intervention was mentioned for this purpose in the AACAP practice parameter. Similar to the NICE guideline, ${ }^{9}$ the AACAP suggests lifestyle modification but with a lack of practical advice for both patients and families. It is the obligation of the clinician to provide both psychoeducation and life skills training to the young people without a standard description and protocol. Hence, the access to recurrence prevention service and quality of facilities are likely to be unequal even within the USA. There is an urgent need to standardize the clinical practice of psychoeducation which has evidence supporting the efficacy of recurrence prevention ${ }^{12}$ and there is a need to develop a well-organized protocol by using these studies as prototypes.

\section{Conclusion}

To date, no study has been published on the prevention and intervention of recurrent depression in adolescents in Thailand. Even though the Thai CPG-MDD-GP is brief and practical, some particular dimensions such as psychosocial intervention for the reduction of both depressive symptoms and recurrence prevention are not mentioned. The Thai CPG-MDD-GP guideline was derived from the guidelines of NICE (2013) and AACAP (2007), but it still lacks details for the family and community in treatment engagement as indicated from both prototypes (Table 2). Hence, further study of prevention of recurrence in depressive adolescents is urgently needed to develop a current guideline to be more holistic and authentic in both the clinical and Thai socio-cultural contexts. Since standard guidelines such as the NICE and AACAP guidelines still highlight treatment considerations on the severity of depressive symptoms, the trajectory of depression and recurrence should be more in focus. Furthermore, the 
upcoming Thai guideline for depression in the adolescent should emphasize family, school, and community-based surveillance with elaboration on the details of recommended psychological interventions for recurrence prevention with a longer period of clinical follow-up (i.e. more than 12-24 months), especially in the high risk groups such as suicide, early onset depression or comorbidity of ADHD.

\section{Acknowledgement}

This article was revised as it was a part of a dissertation, in part fulfillment of the requirements for a Master of Science in Children and Adolescent Mental Health and Psychological Practice, University of Edinburgh. The first author was fully granted her master's degree by the Faculty of Medicine, Prince of Songkla University, Thailand.

\section{References}

1. National Institute of Mental Health. Major depression among adolescents [homepage on the Internet]. Meryland: NIMH; 2013 [cited 2015 May 12]. Available from: http://www.nimh.nih. gov/health/statistics/prevalence/major-depression-amongadolescents.shtml

2. Chaveepojnkamjorn W, Pichainarong N, Adthasangsri V, Sativipawee P, Prasertsong C. Depression and its associated factors among Senior High School students in Nonthaburi Province, Thailand: a cross-sectional study. JPHDC 2017;2: 224-34.

3. Post R. Transduction of psychosocial stress into the neurobiology of recurrent affective disorder. Am J Psychiatry 1992; 149:999-1010.

4. Monroe S, Harkness K. Life stress, the "Kindling" hypothesis, and the recurrence of depression: considerations from a life stress perspective. Psychol Rev 2005;112:417-45.

Table 2 Brief appraisal of the Thai Clinical Practice Guideline of Major Depressive Disorder for General Practitioners (Thai CPG-MDD-GP), National Institute for Health and Care Excellence (NICE), and American Academy of Child and Adolescent Psychiatry (AACAP) guidelines

\begin{tabular}{|c|c|c|c|}
\hline Topic & Thai CPG-MDD-GP ${ }^{8}$ & NICE guideline $^{9}$ & AACAP practice parameter ${ }^{10}$ \\
\hline 1. Health care personnel & $\begin{array}{l}\text { General practitioners in } \\
\text { primary and secondary } \\
\text { care units }\end{array}$ & $\begin{array}{l}\text { Primary health/mental health } \\
\text { care, school and community } \\
\text { personnel }\end{array}$ & $\begin{array}{l}\text { School nurse, pediatrician, } \\
\text { general practitioner, and } \\
\text { mental health care personnel }\end{array}$ \\
\hline $\begin{array}{l}\text { 2. Measurement of depressive } \\
\text { symptoms }\end{array}$ & $\begin{array}{l}\text { 9Q scores } \\
\text { (derived from the Thai } \\
\text { PHQ-9) }\end{array}$ & $\begin{array}{l}\text { Personnel's consideration for } \\
\text { severity (mild, moderate, } \\
\text { severe) }\end{array}$ & $\begin{array}{l}\text { Direct interviewing method (acute, } \\
\text { continuous, maintenance } \\
\text { phase) }\end{array}$ \\
\hline $\begin{array}{l}\text { 3. Psychological intervention } \\
\text { for treatment }\end{array}$ & CBT, IPT-A & $\begin{array}{l}\text { CBT, IPT-A, family therapy } \\
\text { and psychodynamic psycho- } \\
\text { therapy }\end{array}$ & $\begin{array}{l}\text { CBT, supportive therapy, inter- } \\
\text { personal therapy, and psycho- } \\
\text { dynamic psychotherapy }\end{array}$ \\
\hline $\begin{array}{l}\text { 4. Period of time for recurrence } \\
\text { surveillance }\end{array}$ & 12 months & 12-24 months & 12 months \\
\hline $\begin{array}{l}\text { 5. Psychological intervention } \\
\text { for recurrence prevention }\end{array}$ & None & $\begin{array}{l}\text { Suggestion for long-term } \\
\text { lifestyle modification }\end{array}$ & $\begin{array}{l}\text { Life skill training and psycho- } \\
\text { education }\end{array}$ \\
\hline
\end{tabular}

Abbreviations: $\mathrm{PHQ}-9=$ Patient health Questionnaire-9, CBT=cognitive behavioral therapy, IPT-A=interpersonal therapy for depressed adolescents 
5. Herman K, Reinke W, Parkin J, Traylor K, Agarwal G. Childhood depression: rethinking the role of the school. Psychol Sch 2009;46:433-46.

6. Renaud J, Brent D, Baugher M, Birmaher B, Kolko D, Bridge J. Rapid response to psychosocial treatment for adolescent depression: a two-year follow-up. J Am Acad Child Adolesc Psychiatry 1998;37:1184-90.

7. Amattayakong A. Thai mental health database [homepage on the Internet]. Nonthaburi: Department of Mental Health, Ministry of Public Health; 2017 [cited 2015 Jul 22]. Available from: http://www.dmh.go.th/abstract

8. The Excellence Center of Depressive Disorder. Clinical practice guideline of major depressive disorder for general practitioner [homepage on the Internet]. Ubon Ratchatani: Prasrimahabhodi Hospital; 2017 [cited 2015 Jul 20]. Available from: http://www. thaidepression.com

9. National Institute for Health and Care Excellence. Depression in children and young people: identification and management [homepage on the Internet]. London: NICE; 2017 [cited 2015 May 13]. Available from: http://www.nice.org.uk/guidance/cg28

10. Birmaher B, Brent D. Practice parameter for the assessment and treatment of children and adolescents with depressive disorders. J Am Acad Child Adolesc Psychiatry 2007;46:150326.

11. Mahidol University. Faculty of Medicine Siriraj Hospital. Department of Psychiatry. Child and adolescent psychiatric rehabilitation program [homepage on the Internet]. Bangkok:
The University; 2017 [cited 2017 Nov 13]. Available from: http:// www.si.mahidol.ac.th/th/department/psychiatrics/index. asp?pp=newsD\&n_id=153

12. Sanford M, Boyle M, Mccleary L, Miller J, Steele M, Duku E, et al. A pilot study of adjunctive family psychoeducation in adolescent major depression: feasibility and treatment effect. J Am Acad Child Adolesc Psychiatry 2006;45:386-95.

13. Kroenke K, Spitzer R, Williams J. The PHQ-9. J Gen Intern Med 2001;16:606-13.

14. Rohde P, Seeley J, Kaufman N, Clarke G, Stice E. Predicting time to recovery among depressed adolescents treated in two psychosocial group interventions. J Consult Clin Psychol 2006;74:80-8.

15. Birmaher B, Brent D, Kolko D, Baugher M, Bridge J, Holder D, et al. Clinical outcome after short-term psychotherapy for adolescents with major depressive disorder. Arch Gen Psychiatry 2000;57:29.

16. NHS Scotland. CAMH service tiers [homepage on the Internet]. Edinburgh: Healthcare Improvement Scotland: 2017 [cited 2017 Sep 24]. Available from: http://www.icptoolkit. org/child_and_adolescent_pathways/about_icps/camh_ service_tiers.aspx

17. Lewandowski R, Acri M, Hoagwood K, Olfson M, Clarke G, Gardner W, et al. Evidence for the management of adolescent depression. Pediatrics [serial on the Internet]. 2013 Oct [cited 2017 Sep 11]; 132(4). Available from: http://pediatrics. aappublications.org/content/132/4/e996.figures-only 